CERN-TH/2001-031

\title{
Chiral gauge theories revisited
}

\author{
Lectures given at the International School of Subnuclear Physics
}

Erice, 27 August - 5 September 2000

\author{
Martin Lüscher* \\ CERN, Theory Division \\ CH-1211 Geneva 23, Switzerland
}

\section{Contents}

1. Introduction

2. Chiral gauge theories \& the gauge anomaly

3. The regularization problem

4. Weyl fermions from $4+1$ dimensions

5. The Ginsparg-Wilson relation

6. Gauge-invariant lattice regularization of anomaly-free theories

\section{Introduction}

A characteristic feature of the electroweak interactions is that the left- and righthanded components of the fermion fields do not couple to the gauge fields in the same way. The term chiral gauge theory is reserved for field theories of this type, while all other gauge theories (such as QCD) are referred to as vector-like, since the gauge fields only couple to vector currents in this case. At first sight the difference appears to be mathematically insignificant, but it turns out that in many respects chiral

\footnotetext{
* On leave from Deutsches Elektronen-Synchrotron DESY, D-22603 Hamburg, Germany
} 


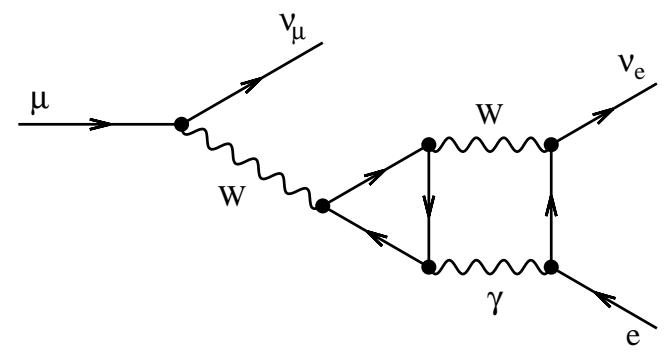

Fig. 1. Feynman diagram contributing to the muon decay at two-loop order of the electroweak interactions. The triangular subdiagram in this example is potentially anomalous and must be treated with care to ensure that gauge invariance is preserved.

gauge theories are much more complicated. Their definition beyond the classical level, for example, is already highly non-trivial and it is in general extremely difficult to obtain any solid information about their non-perturbative properties.

\subsection{Anomalies}

Most of the peculiarities in chiral gauge theories are related to the fact that the gauge symmetry tends to be violated by quantum effects. Whether such anomalies occur or not depends on the gauge group and the fermion multiplet. If they do, the theory probably ceases to be meaningful, since the gauge degrees of freedom are then no longer guaranteed to decouple from the physical modes and unitarity in the physical sector will consequently be lost.

In perturbation theory anomalies can arise from fermion loops with three or more external legs such as the triangle subdiagram in fig. 1. The origin of these anomalies and their topological significance have been completely clarified in the eighties (see refs. [1,2] for comprehensive reviews). Moreover the rigorous work on algebraic renormalization [3-5] showed that there are no further gauge anomalies in perturbation theory, i.e. the gauge symmetry can be preserved to all orders of the expansion if the non-invariant terms cancel at one-loop order.

Additional constraints on the gauge group and the fermion multiplet may have to be imposed to ensure the consistency of the theory also at the non-perturbative level. The $\mathrm{SU}(2)$ gauge theory with a single left-handed fermion in the fundamental representation, for example, has no anomaly in perturbation theory, but there is a non-perturbative global anomaly that ruins the theory [6-8]. While this may be a rather special case, the remark shows how delicate chiral gauge theories are and that there can be unexpected complications beyond perturbation theory. 


\subsection{Fundamental issues in chiral gauge theories}

In the electroweak theory the perturbative anomalies cancel and the perturbation expansion is thus well-defined and consistent to all orders of the gauge couplings. It is, however, hard to overlook the fact that the electroweak theory and chiral gauge theories in general appear to be rather artificial at the quantum level. Whether there is a deeper reason for this is unclear, but a possible explanation could be that chiral gauge theories are merely low-energy effective descriptions of another structure whose mathematical consistency does not depend on accidental cancellations. In such a framework it is conceivable that only the anomaly-free fermion multiplets can decouple from the high-energy degrees of freedom and the electroweak theory would then look a lot more natural than is the case at present.

A second (but not totally unrelated) question is whether chiral gauge theories can be regularized without giving up gauge invariance or other vital properties such as the locality of the theory. In a purely technical sense, such a regularization would provide an example of a well-defined structure that reduces to the desired chiral gauge theory at low energies (i.e. below the cutoff scale). It might also be of some practical importance, since in the absence of a gauge-invariant regularization many non-invariant counterterms must be included in the lagrangian to restore the gauge symmetry after renormalization and removal of the cutoff [3-5,9-15]. As a result the proof of the renormalizability of the theory and the computation of higher-order radiative corrections are far more complicated than in vector-like theories (see ref. [16], for example, and references quoted there).

Beyond perturbation theory, chiral gauge theories are still a largely uncharted territory, and their formulation at this level is in fact already a difficult task. Vectorlike gauge theories are more accessible in this respect, because contrary to chiral theories they can easily be put on the lattice, and numerical simulations then provide a powerful tool to determine their properties. Other approaches, such as the semiclassical approximation, are helpful in studying certain non-perturbative effects but cannot provide a mathematically solid definition of the theory beyond their range of applicability.

\subsection{Scope of the lectures}

Over the last few years significant progress has been made in all areas mentioned above [17-38]. It is now possible, for example, to put anomaly-free chiral gauge theories on the lattice, to all orders of perturbation theory, so that the gauge symmetry is exactly preserved and without having to compromise in any other way [36,37]. Some of these advances grew out of seemingly unrelated lines of research, but most 
of them build on earlier work on chiral gauge theories, such as the descent equations [39-43] and the observation that massive Dirac fermions in $4+1$ dimensions reduce to chiral fermions in 4 dimensions under certain conditions [44-56].

The aim in these lectures is to describe in simple terms some of the key elements of these developments. Since the lectures are intended for non-experts, we shall start with a brief exposition of what everybody should know about chiral gauge theories and the gauge anomaly in perturbation theory. The regularization problem is then described in more detail and a mini-introduction to lattice gauge theory is included to set up basic notations. Some familiarity with this subject is surely helpful but will not be required.

In the central part of the lectures we first discuss how to obtain chiral fermions in 4 dimensions from Dirac fermions in $4+1$ dimensions. This provides an example of a natural mechanism for chiral fermions to arise, and in a few lines it also leads us to the now famous Ginsparg-Wilson relation [57]. Very briefly this identity represents a new form of chiral symmetry that can coexist with a momentum cutoff. In particular, on a space-time lattice it can be taken as the starting point for a general construction of chiral lattice gauge theories, which is the last and most advanced topic that will be addressed in these lectures.

Chiral gauge theories are an old subject, and it is clearly impossible to do justice to all the important contributions that have been made. An excellent introduction to much of the earlier work is provided by the books of Bertlmann on anomalies [2] and of Piguet and Sorella on algebraic renormalization [5]. As far as the development of chiral lattice gauge theories goes, probably the best source of information are the reviews at the yearly lattice conferences [58-61]. Many more references can be found there and also a description of some of the alternative approaches to the problem.

\section{Acknowledgements}

I am indebted to Raymond Stora and Tobias Hurth for discussions on the renormalization of chiral gauge theories and many useful pointers to the literature. Thanks also go to Fred Jegerlehner for sharing his insights on dimensional regularization and to Yoshio Kikukawa and Hiroshi Suzuki with whom I had many enlightening conversations on the topics covered in this course. The hospitality at Erice is legendary,

and I would like to thank Gerard 't Hooft, Gabriele Veneziano and Antonino Zichichi for the opportunity to lecture at this unique place. 


\section{Chiral gauge theories \& the gauge anomaly}

\subsection{Classical theory}

To avoid inessential complications, and since this is common practice in the more mathematical literature on the subject, the theory will be set up in euclidean space. The conventions for the Dirac matrices are

$$
\left\{\gamma_{\mu}, \gamma_{\nu}\right\}=2 \delta_{\mu \nu}, \quad\left(\gamma_{\mu}\right)^{\dagger}=\gamma_{\mu}, \quad \gamma_{5}=\gamma_{0} \gamma_{1} \gamma_{2} \gamma_{3}
$$

and repeated indices are summed over unless stated otherwise.

For simplicity, we shall consider chiral gauge theories with left-handed fermions only and no Higgs fields. If we define the chiral projectors $P_{ \pm}=\frac{1}{2}\left(1 \pm \gamma_{5}\right)$, the fermion and antifermion fields thus satisfy the constraints

$$
P_{-} \psi(x)=\psi(x), \quad \bar{\psi}(x) P_{+}=\bar{\psi}(x) .
$$

Under gauge transformations $\Lambda(x)$, they transform according to

$$
\psi(x) \rightarrow R[\Lambda(x)] \psi(x), \quad \bar{\psi}(x) \rightarrow \bar{\psi}(x) R[\Lambda(x)]^{-1},
$$

where $R$ is some unitary representation of the gauge group $G$. The associated gaugecovariant derivatives

$$
D_{\mu}=\partial_{\mu}+A_{\mu}^{a}(x) R\left(T^{a}\right)
$$

involve the group generators in the representation $R$ and the components $A_{\mu}^{a}(x)$ of the gauge field. The notations here are such that the latter are real, while the group generators are taken to be anti-hermitian.

With these definitions the euclidean action of the theory assumes the form

$$
S[A, \bar{\psi}, \psi]=\int \mathrm{d}^{4} x\left\{\frac{1}{4 g_{0}^{2}} F_{\mu \nu}^{a}(x) F_{\mu \nu}^{a}(x)+\bar{\psi}(x) \gamma_{\mu} D_{\mu} \psi(x)\right\},
$$

where $F_{\mu \nu}^{a}(x)$ denotes the gauge field tensor and $g_{0}$ the gauge coupling. Although we agreed to consider only left-handed fermions, it is now easy to check that charge conjugation maps left- to right-handed fermions in the complex conjugate representation of the gauge group. So if we set

$$
R=R_{\text {left }} \oplus\left(R_{\text {right }}\right)^{*}
$$


the theory defined above is in fact equivalent to one with both left- and righthanded fermions that transform according to the representations $R_{\text {left }}$ and $R_{\text {right }}$ respectively.

\subsection{Gauge anomaly}

From the action (2.5) the Feynman rules can be deduced in the usual way. Gaugefixing is required for this but does not need to be made explicit here, since we shall only consider diagrams without internal gauge field lines. Compared to QCD with massless quarks, an important difference is that the fermion propagator

$$
\langle\psi(x) \bar{\psi}(y)\rangle_{g_{0}=0}=-i \int \frac{\mathrm{d}^{4} p}{(2 \pi)^{4}} \mathrm{e}^{i p(x-y)} \frac{\gamma_{\mu} p_{\mu}}{p^{2}} P_{+}
$$

involves a chiral projector. Apart from this and the fact that the fermion-gaugefield vertices are proportional to $R\left(T^{a}\right)$ instead of the group generator in the quark representation, the Feynman rules are precisely the same.

We now consider the set of fermion one-loop diagrams with $n$ amputated external gauge field lines (see fig. 2). In momentum space the external lines carry in-going momenta $p_{1}, \ldots, p_{n}$, Lorentz indices $\mu_{1}, \ldots, \mu_{n}$ and gauge group indices $a_{1}, \ldots, a_{n}$. The sum of these diagrams is thus some function $V^{(n)}\left(p_{1}, \ldots, p_{n}\right)$ with these many indices. It is not difficult to prove that the associated effective action

$$
\begin{aligned}
S_{\mathrm{eff}}[A]=-\sum_{n=0}^{\infty} \frac{1}{n !} \int & \frac{\mathrm{d}^{4} p_{1}}{(2 \pi)^{4}} \ldots \frac{\mathrm{d}^{4} p_{n}}{(2 \pi)^{4}}(2 \pi)^{4} \delta\left(p_{1}+\ldots+p_{n}\right) \\
& \times V^{(n)}\left(p_{1}, \ldots, p_{n}\right)_{\mu_{1} \ldots \mu_{n}}^{a_{1} \ldots a_{n}} \widetilde{A}_{\mu_{1}}^{a_{1}}\left(p_{1}\right) \ldots \widetilde{A}_{\mu_{n}}^{a_{n}}\left(p_{n}\right)
\end{aligned}
$$

(in which the gauge field plays the rôle of a classical source field) is given by the functional integral

$$
\mathrm{e}^{-S_{\text {eff }}[A]}=\int \mathrm{D}[\psi]_{\text {left }} \mathrm{D}[\bar{\psi}]_{\text {left }} \exp \left\{-\int \mathrm{d}^{4} x \bar{\psi}(x) \gamma_{\mu} D_{\mu} \psi(x)\right\}
$$

over the space of all left-handed fermion and antifermion fields. Indeed, by expanding the integrand on the right-hand side of this equation in powers of the gauge potential, and applying Wick's rule to evaluate the gaussian integral, all products of fermion one-loop diagrams are generated with the proper statistical factors to match the expansion of the left-hand side. 


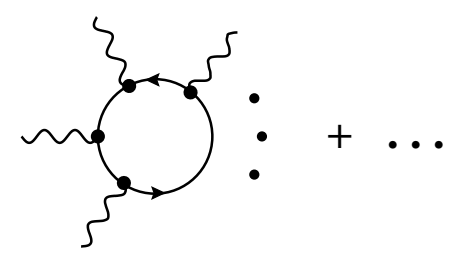

Fig. 2. The vertices $V^{(n)}\left(p_{1}, \ldots, p_{n}\right)$ are equal to the sum of all $(n-1)$ ! fermion one-loop diagrams with $n$ amputated external gauge field lines.

Since all entries in the functional integral respect the gauge symmetry, it seems obvious that the effective action must be a gauge-invariant expression in the gauge potential. However, the fermion one-loop diagrams with less than 5 external lines are ultra-violet-divergent and thus only incompletely defined. Now it can happen that any consistent way to make the diagrams finite necessarily breaks the gauge symmetry of the effective action. In this case we say that there is an anomaly.

The traditional way to cope with ultra-violet divergences is to introduce a regularization and to subtract the divergent terms from the diagrams according to some renormalization scheme. Different regularization and subtraction prescriptions may give different results, but power counting implies that the difference must be a polynomial in the external momenta of a certain degree. This amounts to adding a local term

$$
\Delta S_{\text {eff }}[A]=\int \mathrm{d}^{4} x \Omega(x)
$$

to the effective action, where $\Omega(x)$ is a polynomial in the gauge potential $A_{\mu}^{a}(x)$ and its derivatives of dimension 4 or less. In other words, the effective action in any scheme is the sum of the result obtained with a particular prescription plus such a local term.

At this point an explicit calculation is required to determine the effective action and its gauge transformation properties. The diagrams may be worked out using a Pauli-Villars cutoff, for example, which is defined by substituting

$$
\frac{1}{p^{2}} \rightarrow \frac{1}{p^{2}}-\frac{1}{p^{2}+\Lambda^{2}}
$$

in the fermion propagator (2.7) and taking the cutoff mass $\Lambda$ to infinity. A more elegant computation starts from the observation that the functional integral (2.9) is proportional to the determinant of the Dirac operator $D=\gamma_{\mu} D_{\mu}$. The first-order 
variation of the effective action with respect to the gauge field is thus given by $\dagger$

$$
\delta S_{\text {eff }}=-\operatorname{Tr}\left\{\delta D D^{-1} P_{+}\right\}=-\lim _{\epsilon \rightarrow 0} \int_{\epsilon}^{\infty} \mathrm{d} t \operatorname{Tr}\left\{\delta D D^{\dagger} \mathrm{e}^{-t D D^{\dagger}} P_{+}\right\}
$$

For $\epsilon>0$ the integral on the right-hand side of this equation is finite and provides a regularization of $\delta S_{\text {eff }}$. Differential geometric methods (the heat kernel expansion) may then be applied to study the limit $\epsilon \rightarrow 0$ and to determine the finite part of the effective action in this scheme [62].

Once this is achieved, we may ask whether the now well-defined effective action is invariant under gauge variations

$$
\delta A_{\mu}^{a}(x)=\partial_{\mu} \omega^{a}(x)+f^{a b c} A_{\mu}^{b}(x) \omega^{c}(x)
$$

of the gauge potential (where $\omega^{a}(x)$ denotes an infinitesimal gauge transformation and $f^{a b c}$ the structure constants of the gauge group). As it turns out, the effective action is not invariant in general, but its transformation behaviour can be worked out explicitly and is given by

$$
\begin{aligned}
\delta S_{\text {eff }}= & \frac{i}{192 \pi^{2}} \int \mathrm{d}^{4} x \epsilon_{\mu \nu \rho \sigma} d_{R}^{a b c} \omega^{a}(x)\left\{\partial_{\mu} A_{\nu}^{b}(x) \partial_{\rho} A_{\sigma}^{c}(x)+\frac{1}{2} \partial_{\mu}\left[A_{\nu}^{b}(x) F_{\rho \sigma}^{c}(x)\right]\right\} \\
& +\int \mathrm{d}^{4} x \delta \Omega(x) \\
d_{R}^{a b c}= & 2 i \operatorname{tr}\left\{R\left(T^{a}\right) R\left(T^{b}\right) R\left(T^{c}\right)+(b \leftrightarrow c)\right\}
\end{aligned}
$$

(see ref. [62], for example). Following our discussion above, the variation of an arbitrary local term has been included on the right-hand side of eq. (2.14), and the formula thus holds for any regularization and subtraction scheme.

The algebraic structure of the two terms in eq. (2.14) is such that they cannot cancel each other. Unless $d_{R}^{a b c}$ vanishes identically, gauge invariance is hence violated at one-loop order of perturbation theory. The unphysical longitudinal components of the gauge field must then be expected to couple to the fermions in higher-loop diagrams such as the one shown in fig. 1 . As a consequence unitarity in the physical sector is lost and the theory becomes unusable.

$\dagger$ Here and below the symbol "Tr" denotes a trace in field space, while the lower case "tr" implies a trace over Dirac, colour or flavour indices (depending on the context). 


\subsection{Anomaly-free fermion representations}

The second term in eq. (2.14) can always be cancelled by adding a local counterterm to the gauge-field action, and if $d_{R}^{a b c}$ happens to be equal to zero, the anomaly disappears and the gauge symmetry is preserved. Fermion representations with vanishing $d$-symbol are thus referred to as anomaly-free. In a U(1) theory, for example, there is only one generator, and up to unitary transformations all representations are of the form

$$
R\left(T^{1}\right)=i \times \operatorname{diag}\left(\mathrm{e}_{1}, \ldots, \mathrm{e}_{N}\right), \quad d_{R}^{111}=4 \sum_{\alpha=1}^{N} \mathrm{e}_{\alpha}^{3}
$$

where $N$ denotes the number of fermions and $\mathrm{e}_{\alpha}$ their charges. The anomaly-free representations are then precisely those where the sum of the cubes of the charges vanishes.

Whether a given representation $R$ is anomaly-free or not can often be quickly decided. First note that $d_{R}^{a b c}$ is real, since the symmetrized product of the generators in the trace (2.15) is anti-hermitian. Real and pseudo-real representations are hence always anomaly-free. In particular, chiral theories with gauge group $\mathrm{SU}(2)$ are safe from anomalies, because SU(2) has only such representations.

The groups $\mathrm{SU}(n)$ with $n \geq 3$, on the other hand, have complex representations and at least some of them are not anomaly-free. For any representation $R$ of these groups, we have $d_{R}^{a b c}=c_{R} d^{a b c}$ where $d^{a b c}$ denotes the $d$-symbol in the fundamental representation. The representation is thus anomaly-free if and only if

$$
c_{R}=\operatorname{tr}\left\{R(T)^{3}\right\} / \operatorname{tr}\left\{T^{3}\right\}, \quad T \equiv i \times \operatorname{diag}(1, \ldots, 1,1-n),
$$

vanishes. In the standard $\mathrm{SU}(5)$ grand unified theory [63], for example, the fermions transform according to the representation $\mathbf{5}^{*} \oplus \mathbf{1 0}$, which has $c_{R}=c_{\mathbf{5}^{*}}+c_{\mathbf{1 0}}=0$.

At the level of the Lie algebra, a general compact Lie group decomposes into a product of $\mathrm{U}(1)$ factors and simple groups. Anomaly-free representations $R$ of such a group must obviously reduce to anomaly-free representations of all its factors. Going through Cartan's list of the simple Lie algebras, the only ones that admit totally symmetric invariant tensors of rank 3 are those associated with $\mathrm{SU}(n), n \geq 3$ [64]. The representations of all other simple groups are thus anomaly-free, i.e. only the factors with the Lie algebra of $\mathrm{SU}(n)$ and the abelian factors need to be checked.

Even if the representation is anomaly-free when reduced to any one of the factors of the group, mixed anomalies can still be present, where some components of $d_{R}^{a b c}$ with 
indices belonging to different factors do not vanish. In particular, the components

$$
d_{R}^{a b c}=4 i \operatorname{tr}\{\underbrace{R\left(T^{a}\right) R\left(T^{b}\right)}_{\text {simple factor }} \underbrace{R\left(T^{c}\right)}_{\mathrm{U}(1) \text { factor }}\}
$$

need not be equal to zero and there can also be mixed anomalies between different $\mathrm{U}(1)$ factors (but not between different simple factors).

\section{The regularization problem}

Vector-like theories can be regularized without breaking the gauge symmetry, using dimensional regularization, for example, or by putting them on a lattice. It is clear from the beginning that the situation in the chiral case has to be more complicated, because gauge-invariant regularizations surely can only exist if the fermion multiplet is anomaly-free. In particular, any consistent regularization that preserves the gauge symmetry must refer to the fermion representation $R$.

This simple observation alone implies that none of the widely used regularization schemes can be expected to provide a solution of the problem. The difficulty shows up in various ways, but the no-go theorem was always confirmed and at some point the conclusion was drawn that chiral gauge theories cannot be regularized without breaking the gauge symmetry. In the anomaly-free case, the best one can hope for is then that the symmetry will be restored after renormalization and removal of the regularization [3-5,9-15].

Although a straightforward application of the standard methods does not lead to a gauge-invariant regularization of chiral theories, it is instructive to have a closer look at some of them and to determine what exactly goes wrong. A significant part of the present section is devoted to the lattice regularization, which is a particularly well studied case in this respect.

\subsection{Naive dimensional regularization}

Probably the most economical regularization method that we know of is dimensional regularization [65]. To be able to apply it to chiral gauge theories, the Dirac algebra has to be extended to $d$ dimensions and an unambiguous definition of $\gamma_{5}$ must be supplied. The so-called naive dimensional regularization scheme is characterized by

$$
\left\{\gamma_{\mu}, \gamma_{\nu}\right\}=2 \delta_{\mu \nu}, \quad\left\{\gamma_{5}, \gamma_{\mu}\right\}=0, \quad\left(\gamma_{5}\right)^{2}=1
$$




$$
\delta_{\mu \mu}=d, \quad \operatorname{tr}\{1\}=4,
$$

plus the usual integration rules in $d$ dimensions. This scheme is known to be algebraically consistent (any Feynman diagram with non-exceptional external momenta is assigned a well-defined meromorphic function of $d$ ) and it also preserves the gauge symmetry of the theory.

The prescription nevertheless fails to provide an acceptable regularization of chiral gauge theories, because the rules (3.1),(3.2) together with the requirement of analyticity in $d$ imply

$$
\operatorname{tr}\left\{\gamma_{5} \gamma_{\mu_{1}} \ldots \gamma_{\mu_{2 n}}\right\}=0
$$

for all $n$. It is not difficult to establish this result (appendix A). To fully understand its significance, let us again consider the effective action that we discussed in the previous section.

Fermion one-loop diagrams with $n$ external gauge field lines are proportional to the trace of a product of $2 n$ Dirac matrices and $n$ chiral projectors. Since $\gamma_{5}$ anticommutes with the Dirac matrices, the latter can be combined to a single projector and the trace then assumes the form $\operatorname{tr}\left\{P_{+} \gamma_{\mu_{1}} \ldots \gamma_{\mu_{2 n}}\right\}$. Equation (3.3) says that naive dimensional regularization sets the parity-odd part of the trace to zero. The anomaly is thus avoided, but at the same time we know that the answer is incorrect, because a direct evaluation of the trace in four dimensions (using ordinary Dirac matrices) yields a sum of parity-odd terms proportional to $\epsilon_{\mu \nu \rho \sigma}$ if $n \geq 2$.

\subsection{Regularization with higher-derivative terms}

In general the inclusion of higher-derivative terms in the action has a regularizing effect, because the extra derivatives lead to propagators that decrease more rapidly at large momenta [66-69]. The transverse part of the gauge field propagator, for example, decays like $\left(p^{2}\right)^{-2}$ at momenta $p$ much greater than $\Lambda$ if the term

$$
\frac{1}{\Lambda^{2}} \int \mathrm{d}^{4} x D_{\mu} F_{\nu \rho}^{a}(x) D_{\mu} F_{\nu \rho}^{a}(x)
$$

is added to the action. The mass $\Lambda$ then plays the rôle of an ultra-violet cutoff that is to be sent to infinity at the end of the calculation.

In this strictly four-dimensional approach, the definition of the Dirac matrices is not an issue, but the question of whether the gauge symmetry can be preserved needs to be carefully discussed (even in the pure gauge theory). First note that higher-derivative terms have to be included to suppress the propagators of the ghost 
fields and the longitudinal components of the gauge field at large momenta after fixing the gauge. This can be done so that the total gauge-fixed action is invariant under the Becchi-Rouet-Stora (BRS) transformation [3-5], and the gauge symmetry is then guaranteed to be preserved at the level of the physical amplitudes.

Power-counting now shows, however, that no matter which higher-derivative terms are added, some one-loop diagrams always remain unregularized, because terms like (3.4) not only modify the propagators but also give rise to additional vertices. These vertices are required to ensure the gauge invariance of the regularized theory, and while they are suppressed by inverse powers of the cutoff $\Lambda$, their insertion increases the degree of divergence of the diagrams. As a result some of these diagrams have positive degree.

Further regularization prescriptions are thus needed to make all diagrams finite. In refs. [66-69], for example, it has been proposed to include a set of Pauli-Villars ghost fields with properly chosen couplings to the gauge field. Such hybrid regularizations are delicate, and it is not easy to prove the correctness of the procedure. Some of these schemes are in fact known to yield wrong results [70,71], while others require intermediate dimensional regularization [72] or include non-local terms in the action [73]. Adding higher-derivative terms is, therefore, still not a very transparent regularization method, not even in vector-like theories (see, however, ref. [74]).

\subsection{Lattice gauge theory}

The lattice formulation of quantum field theories provides a good starting point for non-perturbative studies, and this is no doubt the principal reason why lattice field theory continues to be a popular research topic for now more than 25 years. In the present context we are interested in perturbation theory, where the lattice makes all diagrams manifestly finite.

Lattice field theories are usually set up on a hypercubic lattice with spacing $a$ (see fig. 3). A fermion field $\psi(x)$, for example, is then simply an assignment of a Dirac spinor to each lattice point

$$
x=a\left(n_{0}, n_{1}, n_{2}, n_{3}\right), \quad n_{\mu} \in \mathbb{Z} .
$$

The Fourier representation of any such field,

$$
\psi(x)=\int_{-\pi / a}^{\pi / a} \frac{\mathrm{d}^{4} p}{(2 \pi)^{4}} \mathrm{e}^{i p x} \widetilde{\psi}(p),
$$

involves an integration over momenta in a bounded region only (the Brillouin zone), and lattice field theories thus have a built-in momentum cutoff of order $1 / a$. 

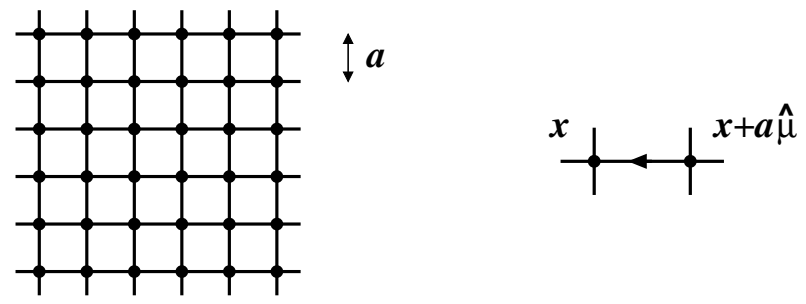

Fig. 3. In lattice gauge theory the fermion fields $\psi(x)$ reside on the points $x$ of a regular hypercubic lattice with spacing $a$ and the gauge field variables $U(x, \mu)$ on the directed links $(x, x+a \hat{\mu})$ of the lattice (expanded view on the right).

On the lattice the forward and backward difference operators

$$
\begin{aligned}
& \partial_{\mu} \psi(x)=\{\psi(x+a \hat{\mu})-\psi(x)\} / a, \\
& \partial_{\mu}^{*} \psi(x)=\{\psi(x)-\psi(x-a \hat{\mu})\} / a,
\end{aligned}
$$

(where $\hat{\mu}$ denotes the unit vector in direction $\mu$ ) can be taken as substitutes for the partial differential operators in the continuum theory. It is useful to introduce them both, because $\partial_{\mu}^{*}$ is equal to minus the adjoint of $\partial_{\mu}$ and vice versa. The lattice laplacian, for example, can then be compactly written as $\partial_{\mu}^{*} \partial_{\mu}$.

Lattice Dirac operators that reduce to the continuum Dirac operator in the limit $a \rightarrow 0$ are now also easily constructed. Partly because of its simplicity, the expression proposed by Wilson in 1974 [75],

$$
D_{\mathrm{w}}=\frac{1}{2}\left\{\gamma_{\mu}\left(\partial_{\mu}^{*}+\partial_{\mu}\right)-a \partial_{\mu}^{*} \partial_{\mu}\right\}
$$

is still widely used today, but there are many other acceptable lattice Dirac operators that all lead to the same continuum limit (the significance of the second term in the definition of $D_{\mathrm{w}}$ will be clarified in the next subsection).

In the continuum theory, gauge-covariant differentiation requires the introduction of gauge potentials with the appropriate transformation behaviour. Lattice gauge fields serve exactly the same purpose, except that here we are dealing with difference instead of differential operators. Explicitly, if we assume that the gauge group acts on lattice fermion fields in the obvious way [eq. (2.3)], and if $U(x, \mu)$ is a lattice field with values in the gauge group, which transforms according to

$$
U(x, \mu) \rightarrow \Lambda(x) U(x, \mu) \Lambda(x+a \hat{\mu})^{-1},
$$


it is trivial to check that the difference operators

$$
\begin{aligned}
& \nabla_{\mu} \psi(x)=\{R[U(x, \mu)] \psi(x+a \hat{\mu})-\psi(x)\} / a, \\
& \nabla_{\mu}^{*} \psi(x)=\left\{\psi(x)-R[U(x-a \hat{\mu}, \mu)]^{-1} \psi(x-a \hat{\mu})\right\} / a,
\end{aligned}
$$

are gauge-covariant (cf. fig. 3). On the lattice, gauge fields are thus represented by group-valued fields $U(x, \mu)$ rather than vector fields with values in the Lie algebra of the gauge group. This seems a bit strange at first sight, but should not be given too much weight, because the difference is mainly a matter of notation. In perturbation theory, for example, the parametrization

$$
U(x, \mu)=\exp \left\{a A_{\mu}^{a}(x) T^{a}\right\}=1+a A_{\mu}^{a}(x) T^{a}+\ldots
$$

is usually employed, which leads again to a description in terms of a gauge potential $A_{\mu}^{a}(x)$. The covariant difference operators $\nabla_{\mu}$ and $\nabla_{\mu}^{*}$ are then also easily seen to converge to the covariant differential operator $D_{\mu}$ in the limit $a \rightarrow 0$.

It is now obvious that the lattice fermion action

$$
S_{\mathrm{F}}[U, \bar{\psi}, \psi]=a^{4} \sum_{x} \bar{\psi}(x) D_{\mathrm{w}} \psi(x)
$$

preserves the gauge symmetry if the ordinary difference operators in the definition (3.9) of the Wilson-Dirac operator are replaced by covariant ones. Invariant gauge field actions are also not difficult to construct [75-77] and the bottom line is then that vector-like theories such as QCD can be put on the lattice without breaking the gauge symmetry or running into any other fundamental difficulty. The lattice formulation of these theories has in fact long been shown to provide a completely consistent regularization to all orders of perturbation theory. In particular, gaugefixing is a rigorous procedure in this framework, and the existence of the continuum limit has been established using the Reisz power-counting theorem and the BRS symmetry [78-80].

\subsection{The Nielsen-Ninomiya no-go theorem}

At this point it seems that chiral lattice gauge theories can be obtained simply by imposing the constraints (2.2) on the fermion and antifermion fields. To understand why this is not so, it suffices to consider the free fermion theory. First note that

$$
D_{\mathrm{w}} \mathrm{e}^{i p x} u=\left\{i \gamma_{\mu} \stackrel{\circ}{p}_{\mu}+\frac{1}{2} a \hat{p}^{2}\right\} \mathrm{e}^{i p x} u,
$$




$$
\stackrel{\circ}{p}_{\mu}=(1 / a) \sin \left(a p_{\mu}\right), \quad \hat{p}_{\mu}=(2 / a) \sin \left(a p_{\mu} / 2\right),
$$

for any four-momentum $p$ and Dirac spinor $u$. The fermion propagator (which coincides with the Green function of the Wilson-Dirac operator) is thus given by

$$
\langle\psi(x) \bar{\psi}(y)\rangle=-i \int_{-\pi / a}^{\pi / a} \frac{\mathrm{d}^{4} p}{(2 \pi)^{4}} \mathrm{e}^{i p(x-y)} \frac{\gamma_{\mu} \stackrel{\circ}{\mu}_{\mu}+i \frac{1}{2} a \hat{p}^{2}}{\grave{p}^{2}+\frac{1}{4} a^{2}\left(\hat{p}^{2}\right)^{2}}
$$

In particular, the propagator has no singularities in momentum space other than the expected one-particle pole at $p=0$.

Now if we impose the chiral constraints (2.2), the part of the Wilson-Dirac operator that survives in the fermion action (3.14) is

$$
P_{+} D_{\mathrm{w}} P_{-}=\frac{1}{2} P_{+} \gamma_{\mu}\left(\partial_{\mu}^{*}+\partial_{\mu}\right)
$$

In momentum space the denominator of the chiral propagator is hence equal to $\grave{p}^{2}$ and not $\dot{p}^{2}+\frac{1}{4} a^{2}\left(\hat{p}^{2}\right)^{2}$ as in the Dirac case. As a consequence there are now poles at all momenta with components $p_{\mu} \in\{0, \pm \pi / a\}$, and these poles effectively describe separate fermion species. If the fermion field is coupled to an external gauge field, for example, the effective action turns out to coincide, in the continuum limit, with the result expected for a theory with these many fermion flavours.

The fermion doubling problem (as it is called in the literature) is not specific to any particular lattice formulation. The Nielsen-Ninomiya no-go theorem $[81,82]$ in fact asserts that the chiral projection with the projectors $P_{ \pm}$always leads to unphysical poles in the fermion propagator if the chosen lattice Dirac operator is local. Having more fermions than intended or giving up the locality of the theory is clearly unacceptable. There were many attempts to circumvent this difficulty, but it is only in the last two years that a solution has finally been found. A particularly appealing approach to this solution starts from fermions in $4+1$ dimensions, which is the topic of the next section.

Appendix A. Proof of eq. (3.3)

After inserting the identity $\gamma_{\mu} \gamma_{\mu}=d$ and cycling one of the factors $\gamma_{\mu}$ around the trace, using the anticommutation rules (3.1), an equation of the form

$$
(d-2 n) \operatorname{tr}\left\{\gamma_{5} \gamma_{\mu_{1}} \ldots \gamma_{\mu_{2 n}}\right\}+\ldots=0
$$

is obtained, where the ellipses stand for a sum of traces with $2 n-2$ Dirac matrices. In particular, for $n=0$ the algebra yields $d \operatorname{tr}\left\{\gamma_{5}\right\}=0$ and thus $\operatorname{tr}\left\{\gamma_{5}\right\}=0$. Equation (3.3) now follows from eq. (A.1) by induction over $n$. 
Note that we cannot escape the argument by assigning a non-zero value to the traces at $d=2 n$ only, because in a dimensional regularization scheme the Feynman integrals must be analytic functions of $d$ and such singular solutions of the recursion (A.1) are hence not acceptable.

\section{Weyl fermions from $4+1$ dimensions}

The descent equations [39-43] were probably the first instance where a connection between chiral fermions and field theories in higher dimensions was made. At the time this seemed to be a purely algebraic observation, relating the Chern character in six dimensions via the Chern-Simons density in five dimensions to the gauge anomaly. The relationship in fact goes far beyond the formal level [44-56], and while the discussion in the present section is rather limited in scope, it can be regarded as a first step in this direction.

\subsection{Domain wall fermions}

Since the fifth dimension is going to play a special rôle, we do not employ a covariant notation, i.e. the extra coordinate will be denoted by $s$ and Lorentz indices $\mu, \nu, \ldots$ label the four physical directions as before. In the presence of a scalar background field $\phi(s)$, the Dirac operator in $4+1$ dimensions is then given by

$$
D_{5}=\gamma_{\mu} \partial_{\mu}+\gamma_{5} \partial_{s}-\phi(s)
$$

The question of where the background field comes from will not concern us here, and we shall simply assume that it has the shape of a step function of height $M$ and width $1 / M$ (see fig. 4). Such a background field defines a domain wall separating the half-spaces $s<0$ and $s>0$ from each other.

We now show that the domain wall affects the physical properties of the fermions in an interesting way $[44,45]$. Fermion fields in $4+1$ dimensions with energy $E$ and momentum $\mathbf{p}$ in 4 dimensions are of the form

$$
\chi(x, s)=\mathrm{e}^{i p x} u(s), \quad p=(i E, \mathbf{p})
$$

(recall that we are using a euclidean metric, where the energy components of physical four-momenta are purely imaginary). The Dirac equation $D_{5} \chi(x, s)=0$ then 


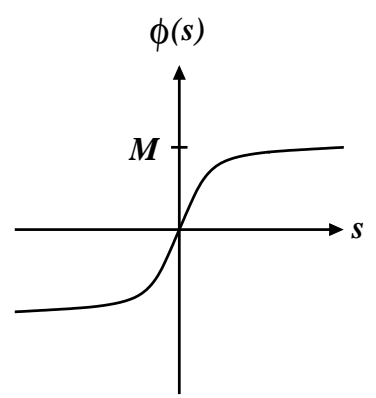

Fig. 4. Qualitative shape of the background field $\phi(s)$. The prototype of such a function is $\phi(s)=M \tanh (M s)$, but its analytic form will not be needed here.

becomes

$$
\left\{\gamma_{5} \partial_{s}-\phi(s)\right\} u(s)=-i \gamma_{\mu} p_{\mu} u(s)
$$

and after multiplication from the left with $-i \gamma_{\mu} p_{\mu}$, this leads to the equation

$$
\begin{aligned}
& \left\{-\partial_{s}^{2}+V(s)\right\} u(s)=m^{2} u(s), \\
& V(s)=\gamma_{5} \partial_{s} \phi(s)+\phi(s)^{2}, \quad m^{2}=E^{2}-\mathbf{p}^{2} .
\end{aligned}
$$

The possible fermion masses $m$ are thus determined by the eigenvalues of a certain differential operator. In other words, from the point of view of the four-dimensional world, the presence of the extra dimension results in a tower of fermions with these masses.

The operator on the left-hand side of eq. (4.4) commutes with $\gamma_{5}$ and its eigenfunctions may hence be assumed to have definite chirality. We now distinguish three cases.

(a) Continuous spectrum. Independently of the chirality, the asymptotic value of the potential $V(s)$ at large $|s|$ is equal to $M^{2}$, and the spectrum of the operator thus includes the half-line $m^{2} \geq M^{2}$ (see fig. 5).

(b) Discrete spectrum. Eigenfunctions with eigenvalues below $M^{2}$ decay exponentially and this part of the mass spectrum is thus purely discrete. Negative eigenvalues (tachyons) are excluded, since

$$
-\partial_{s}^{2}+V(s)=\left\{-\gamma_{5} \partial_{s}+\phi(s)\right\}^{\dagger}\left\{-\gamma_{5} \partial_{s}+\phi(s)\right\}
$$




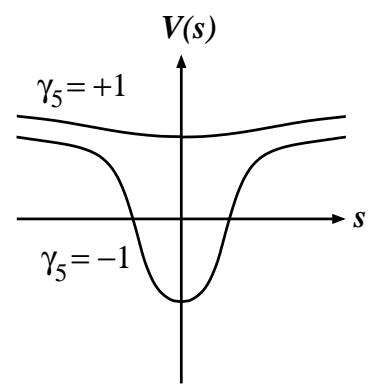

Fig. 5. For negative chirality, the potential $V(s)$ has a well at $s=0$ in which the massless mode is trapped. The right-handed modes, on the other hand, are always heavy independently of whether they are bound to the domain wall or not.

is a non-negative operator. Moreover, all non-zero mass values $m$ must be of order $M$, since there is no other characteristic scale around.

(c) Massless modes. In this case the Dirac equation reduces to

$$
\left\{-\gamma_{5} \partial_{s}+\phi(s)\right\} u(s)=0, \quad \gamma_{\mu} p_{\mu} u(s)=0,
$$

from which we infer that

$$
u(s)=\exp \left\{ \pm \int_{0}^{s} \mathrm{~d} t \phi(t)\right\} v, \quad \gamma_{\mu} p_{\mu} v=0, \quad P_{ \pm} v=v
$$

While the positive chirality solution grows exponentially at large $|s|$, the left-handed mode is normalizable and $m^{2}=0$ thus belongs to the discrete mass spectrum.

To sum up we have found that all fermion modes, except for one, have mass $m$ of order $M$ or larger. The massless mode is left-handed and its wave function [which is given by eqs. (4.2),(4.8)] falls off exponentially in the extra dimension. At energies $E$ far below $M$, the theory thus describes a single left-handed chiral fermion that propagates along the domain wall. Effectively a dimensional reduction from $4+1$ to 4 dimensions is taking place, since it requires an energy proportional to $M$ or larger to excite the higher fermion modes $[44,45]$.

\subsection{Fermion propagator}

An attractive feature of this mechanism is its stability against various changes of the set-up. In particular, the precise form of the shape function $\phi(s)$ is irrelevant, and we may in fact replace the domain wall through a boundary with Dirichlet boundary 
conditions. The fermion propagator can then be worked out explicitly and provides further insight into what dimensional reduction means in the present context.

So let us consider Dirac fields $\chi(x, s)$ in the half-space $s \geq 0$ that satisfy

$$
\left.P_{+} \chi(x, s)\right|_{s=0}=0 \text {. }
$$

In this formulation a background field is no longer required, and for the Dirac operator we can simply take

$$
D_{5}=D_{4}+\gamma_{5} \partial_{s}-M, \quad D_{4}=\gamma_{\mu} \partial_{\mu}
$$

The spectrum of fermion masses is then as before, with a left-handed Weyl fermion that moves along the boundary at $s=0$.

In $4+1$ dimensions the fermion propagator is defined by

$$
\left.D_{5} G(x, s ; y, t)\right|_{s, t>0}=\delta(x-y) \delta(s-t),\left.\quad P_{+} G(x, s ; y, t)\right|_{s=0}=0 .
$$

Since $D_{4}$ does not depend on the extra coordinate, it is straightforward to solve these equations (appendix). In particular, for $x \neq y$ the result

$$
\left.G(x, s ; y, t)\right|_{s=t=0}=2 M \times P_{-} S(x, y) P_{+}
$$

is obtained, where $S(x, y)$ denotes the Green function of the operator

$$
D=M+\left(D_{4}-M\right)\left[1-\left(D_{4} / M\right)^{2}\right]^{-1 / 2}
$$

(that acts on Dirac fields in four dimensions). The inverse square root in this formula is defined in the obvious way, taking into account the fact that the operator in square brackets is hermitian and bounded from below by 1 .

The propagator (4.12) describes the fermion propagation along the boundary of the five-dimensional world and should reflect the presence of the massless mode. This is indeed the case, since at four-momenta far below $M$, the operator $D$ is given by

$$
D=D_{4}\left\{1-\frac{D_{4}}{2 M}+\ldots\right\}
$$

i.e. it coincides with the four-dimensional Dirac operator up to terms of order $1 / M$. In particular, the right-hand side of eq. (4.12) is equal to the propagator of a free 
left-handed fermion in this kinematical regime. The reduction from Dirac fermions in five dimensions to Weyl fermions in four dimensions thus also works out at the level of the underlying field theory.

Appendix B. Analytic formula for $G(x, s ; y, t)$

To simplify the notation, we interpret the propagator at fixed $s$ and $t$ as an operator $\mathcal{G}(s, t)$ that acts on Dirac fields $\psi(x)$ in four dimensions according to

$$
\mathcal{G}(s, t) \psi(x)=\int \mathrm{d}^{4} y G(x, s ; y, t) \psi(y)
$$

In operator form, the equations that need to be solved are

$$
\left.D_{5} \mathcal{G}(s, t)\right|_{s, t>0}=\delta(s-t),\left.\quad P_{+} \mathcal{G}(s, t)\right|_{s=0}=0,
$$

and this can be done elegantly in terms of the hermitian operators

$$
Q=\gamma_{5}\left(M-D_{4}\right), \quad \hat{P}_{ \pm}=\frac{1}{2}\left\{1 \pm Q\left[Q^{2}\right]^{-1 / 2}\right\}
$$

The latter are just the projectors to the subspaces of eigenvectors of $Q$ with positive and negative eigenvalues. Note that the definition of these subspaces is unambiguous, since all eigenvalues of $Q$ have absolute magnitude greater than or equal to $M$.

The claim is now that the operator

$$
\mathcal{G}(s, t)=\left\{\mathrm{e}^{(s-t) Q}\left[\theta(s-t) \hat{P}_{-}-\theta(t-s) \hat{P}_{+}\right]+\mathrm{e}^{s Q} \hat{P}_{-} \frac{2 M}{D} \hat{P}_{+} \mathrm{e}^{-t Q}\right\} \gamma_{5}
$$

has all the required properties. One of these is that the expression should go to zero if $|s-t|$ becomes large, which is the case since the exponentials are multiplied with the appropriate product of step functions and projectors $\hat{P}_{ \pm}$. Using $D_{5}=\gamma_{5}\left(\partial_{s}-Q\right)$, it is also trivial to show that the Dirac equation holds. Finally, starting from the definitions (4.13) and (4.17), the identity

$$
2 M P_{+} \hat{P}_{-}=P_{+} D
$$

may be deduced, and this immediately implies that the operator (B.4) satisfies the boundary condition. 


\section{The Ginsparg-Wilson relation}

\subsection{Kaplan's observation}

As already mentioned, the occurrence of a massless fermion mode in the presence of a domain wall is a generic effect. In 1992 Kaplan noted that the massless mode persists even if the five-dimensional space is replaced by a lattice [46]. It is natural to set $M=1 / a$ in this context (where $a$ denotes the lattice spacing), and the masses of the heavy modes are then of the order of the momentum cutoff or larger.

An inaccurate but often quoted form of the Nielsen-Ninomiya theorem states that it is not possible to have a single Weyl fermion on a four-dimensional lattice. This was, however, precisely what Kaplan obtained. In particular, he showed that there was no doubling of fermion species in four dimensions if the Wilson-Dirac operator was used in five dimensions (cf. subsect. 3.3).

The resolution of this puzzle basically is that chiral symmetry can be realized in different ways. To make this a bit more concrete, let us go back to the continuum theory studied in the previous section. As explained there, the fermion propagator (4.12) along the domain wall coincides with the left-handed components of the Green function of a modified Dirac operator $D$ [eq. (4.13)]. Now the surprise is that $D$ does not anticommute with $\gamma_{5}$ and instead satisfies the equation

$$
\gamma_{5} D+D \gamma_{5}=\frac{1}{M} D \gamma_{5} D
$$

This relation first appeared in 1982 in a paper of Ginsparg and Wilson [57] on a completely different topic (the block-spin renormalization group in lattice QCD). At the time it was considered to represent some sort of remnant chiral symmetry, but there is in fact much more behind this remarkable identity.

\subsection{Significance of the Ginsparg-Wilson relation}

In the following paragraphs we only use the Ginsparg-Wilson relation (5.1) and the hermiticity property $D^{\dagger}=\gamma_{5} D \gamma_{5}$. Although we are in the continuum theory here, the results that we shall obtain are of a very general nature and can easily be carried over to the lattice (as we shall see later in this section).

(a) Propagator. In terms of the Green function $S(x, y)$ of $D$, the Ginsparg-Wilson relation assumes the form

$$
S(x, y) \gamma_{5}+\gamma_{5} S(x, y)=\frac{1}{M} \gamma_{5} \delta(x-y)
$$


The propagator is hence chirally invariant at all non-zero distances. In particular, the residue of the particle pole in momentum space anticommutes with $\gamma_{5}$ and we can say, therefore, that the Ginsparg-Wilson relation implies chiral invariance on the mass shell.

(b) Off-shell chiral symmetry. If we define the fermion action as usual,

$$
S_{\mathrm{F}}[\bar{\psi}, \psi]=\int \mathrm{d}^{4} x \bar{\psi}(x) D \psi(x)
$$

it is trivial to check that the infinitesimal transformation

$$
\begin{aligned}
& \psi \rightarrow \psi+\epsilon \gamma_{5}(1-D / M) \psi, \\
& \bar{\psi} \rightarrow \bar{\psi}+\epsilon \bar{\psi} \gamma_{5}
\end{aligned}
$$

leaves the action invariant to first order in $\epsilon[20]$. At momenta far below $M$, this transformation reduces to an ordinary chiral rotation, and it can hence be regarded as an unusual but exact realization of chiral symmetry. Note incidentally that the fermion and antifermion fields can be transformed independently of each other in euclidean space. Eventually they are both integrated over in the functional integral, and the transformation (5.4),(5.5) is then just a substitution of integration variables.

(c) Weyl fermions. Now that we have an exact chiral symmetry, it is not difficult to pass to chiral fermions. First note that the operator $\hat{\gamma}_{5}=\gamma_{5}(1-D / M)$ satisfies

$$
\left(\hat{\gamma}_{5}\right)^{\dagger}=\hat{\gamma}_{5}, \quad\left(\hat{\gamma}_{5}\right)^{2}=1, \quad \gamma_{5} D=-D \hat{\gamma}_{5}
$$

The fermion action thus splits into left- and right-handed parts, if the chiral projectors for fermion and antifermion fields are defined through [28,29,33]

$$
\hat{P}_{ \pm}=\frac{1}{2}\left(1 \pm \hat{\gamma}_{5}\right), \quad P_{ \pm}=\frac{1}{2}\left(1 \pm \gamma_{5}\right)
$$

respectively. It may seem strange not to use the same projectors for both fields, but in euclidean space this is certainly permissible and probably also unavoidable in view of the transformation law (5.4), (5.5).

We can now eliminate the right-handed components by imposing the constraints

$$
\hat{P}_{-} \psi=\psi, \quad \bar{\psi} P_{+}=\bar{\psi}
$$


The associated propagator is the inverse of the projected operator $P_{+} D \hat{P}_{-}$in the subspace of left-handed fields and is thus given by

$$
\hat{P}_{-} S(x, y) P_{+}=P_{-} S(x, y) P_{+}+\frac{1}{2 M} P_{+} \delta(x-y),
$$

where $S(x, y)$ denotes the Green function of $D$ as before. Up to the contact term and an uninteresting normalization factor, this expression coincides with the propagator (4.12) of the fermion field in $4+1$ dimensions along the domain wall. In other words, at low energies, domain wall fermions reduce to a chiral theory in four dimensions in which the chiral symmetry is realized through the Ginsparg-Wilson relation [49].

\subsection{Lattice fermions with exact chiral symmetry}

Domain wall fermions on a five-dimensional lattice can be studied in essentially the same way as in the continuum theory. In particular, the domain wall may again be replaced by a boundary with Dirichlet boundary conditions. A further technical simplification is achieved by taking the lattice spacing in the extra dimension to zero while keeping the spacing $a$ in four dimensions fixed. The fermion propagator is then obtained as before (subsect. 4.2). If we set

$$
D_{5}=D_{\mathrm{w}}+\gamma_{5} \partial_{s}-M, \quad M=1 / a
$$

for example, where $D_{\mathrm{w}}$ denotes the Wilson-Dirac operator (3.9), the boundary value of the propagator is still given by eq. (4.12), with $S(x, y)$ the Green function of the operator [18]

$$
D=\frac{1}{a}\left\{1-\left(1-a D_{\mathrm{w}}\right)\left[\left(1-a D_{\mathrm{w}}\right)^{\dagger}\left(1-a D_{\mathrm{w}}\right)\right]^{-1 / 2}\right\}
$$

Up to lattice corrections of order $a$, this operator coincides with $D_{\mathrm{w}}$, and it may hence be regarded as another possible discretization of the continuum Dirac operator. Moreover, it satisfies the Ginsparg-Wilson relation,

$$
\gamma_{5} D+D \gamma_{5}=a D \gamma_{5} D
$$

and also the hermiticity condition $D^{\dagger}=\gamma_{5} D \gamma_{5}$. From our discussion in the previous subsection, we thus conclude that lattice fermions in four dimensions with lattice Dirac operator $D$ preserve chiral symmetry in the form of the infinitesimal transformation (5.4),(5.5). In particular, as explained above, they can be split into leftand right-handed components in a consistent way. 
Although this is not in conflict with the precise statement of the Nielsen-Ninomiya theorem, some doubt may remain that we have indeed managed to avoid the doubling problem. The following remarks should make it clear that there is really nothing to complain about here.

(a) Unphysical poles. From the definition (5.11) and eqs. (3.15),(3.16) it is straightforward to deduce that $D$ is given by

$$
D=\frac{1}{a}\left\{1-\left[1-\frac{1}{2} a^{2} \hat{p}^{2}-i a \gamma_{\mu} \stackrel{\circ}{\mu}_{\mu}\right]\left[1+\frac{1}{2} a^{4} \sum_{\mu<\nu} \hat{p}_{\mu}^{2} \hat{p}_{\nu}^{2}\right]^{-1 / 2}\right\}
$$

in momentum space. This expression is analytic in the whole range $\left|p_{\mu}\right| \leq \pi / a$ of lattice momenta. It can also be shown to be invertible at all these momenta except at $p=0$ where

$$
D=i \gamma_{\mu} p_{\mu}+\mathrm{O}\left(a p^{2}\right)
$$

In particular, the propagator $1 / D$ has no unphysical poles.

(b) Locality. In position space the action of $D$ on an arbitrary fermion field $\psi(x)$ is given by a kernel $D(x, y)$ through

$$
D \psi(x)=a^{4} \sum_{y} D(x, y) \psi(y)
$$

The kernel is translation-invariant and equal to the Fourier transform of the righthand side of eq. (5.13). Starting from this representation, it is possible to derive a bound of the form [21]

$$
\|D(x, y)\| \leq C \mathrm{e}^{-\|x-y\| / \varrho},
$$

which shows that $D$ is a local operator with localization range $\varrho$. From the point of view of the continuum limit, this is as good as strict locality, since the range $\varrho$ is estimated to be no more than a few lattice spacings.

(c) Unitarity. As is generally true in a free field theory, all the information about the underlying Hilbert space of physical states and the energy-momentum spectrum can be retrieved from the propagator $S(x, y)$ (which represents $1 / D$ in position space). In particular, negative norm or complex energy states are excluded if and only if the propagator admits a Källén-Lehmann representation

$$
\left.S(x, y)\right|_{x_{0}>y_{0}}=\int_{0}^{\infty} \mathrm{d} E \int_{-\pi / a}^{\pi / a} \frac{\mathrm{d}^{3} \mathbf{p}}{(2 \pi)^{3}} \sigma(E, \mathbf{p}) \mathrm{e}^{-E\left(x_{0}-y_{0}\right)+i \mathbf{p}(\mathbf{x}-\mathbf{y})}
$$


with non-negative spectral density, viz.

$$
\bar{u} \sigma(E, \mathbf{p}) u \geq 0, \quad \bar{u}=u^{\dagger} \gamma_{0},
$$

for all Dirac spinors $u$. The expression (5.13) for the Dirac operator in momentum space looks rather complicated and it seems unlikely that $S(x, y)$ has such a representation, but a lengthy exercise in complex contour integration shows this to be the case. Unitarity is hence respected for any value of the lattice spacing.

\subsection{Adding gauge fields}

So far our discussion of domain wall fermions and the Ginsparg-Wilson relation has been limited to free fermions. Gauge fields may now easily be included, however, by replacing the free Dirac operator in four dimensions by the gauge-covariant operator. Most of the formulae that we have obtained then remain valid without modification. The fermion propagator in $4+1$ dimensions, for example, is still given by eq. (B.4), and the dimensional reduction thus works out as before.

In the presence of the gauge field, eq. (5.11) defines a gauge-covariant lattice Dirac operator that satisfies the Ginsparg-Wilson relation (5.12). Using this operator, we can build a version of lattice QCD in which chiral symmetry is preserved. The infinitesimal chiral rotations

$$
\psi \rightarrow \psi+\epsilon \lambda^{a} \hat{\gamma}_{5} \psi, \quad \bar{\psi} \rightarrow \bar{\psi}+\epsilon \bar{\psi} \gamma_{5} \lambda^{a}
$$

may include a flavour matrix $\lambda^{a}$ in this case, and the lattice action

$$
S_{\mathrm{F}}[U, \bar{\psi}, \psi]=a^{4} \sum_{x} \bar{\psi}(x) D \psi(x)
$$

is then invariant under the full chiral flavour group.

This seems to contradict the fact that the flavour-singlet axial symmetry in QCD with $N_{\mathrm{f}}$ massless quarks is known to be broken by the axial anomaly. However, symmetry transformations in quantum field theory must not only preserve the action but also the integration measure in the functional integral. In lattice QCD the fermion and antifermion integration measures are given by

$$
\mathrm{D}[\psi]=\prod_{x, \alpha} \mathrm{d} \psi_{\alpha}(x), \quad \mathrm{D}[\bar{\psi}]=\prod_{x, \alpha} \mathrm{d} \bar{\psi}_{\alpha}(x),
$$


where $\alpha$ collectively denotes the Dirac, colour and flavour indices of the fields. To first order in $\epsilon$, these measures transform according to

$$
\mathrm{D}[\psi] \rightarrow\left[1-\epsilon \operatorname{Tr}\left\{\lambda^{a} \hat{\gamma}_{5}\right\}\right] \mathrm{D}[\psi], \quad \mathrm{D}[\bar{\psi}] \rightarrow \mathrm{D}[\bar{\psi}]
$$

under the chiral rotations (5.19).

Since $\hat{\gamma}_{5}$ is trivial in flavour space, the jacobian in eq. (5.22) is equal to unity if $\operatorname{tr}\left\{\lambda^{a}\right\}=0$. The flavoured transformations are hence exact symmetries of the theory. This is not so in the flavour-singlet case, where [19-27]

$$
\begin{aligned}
\operatorname{Tr}\left\{\hat{\gamma}_{5}\right\} & =-a^{4} \sum_{x} \operatorname{tr}\left\{\gamma_{5} a D(x, x)\right\} \\
& \underset{a \rightarrow 0}{\sim}-\frac{N_{\mathrm{f}}}{32 \pi^{2}} \int \mathrm{d}^{4} x \epsilon_{\mu \nu \rho \sigma} F_{\mu \nu}^{a}(x) F_{\rho \sigma}^{a}(x) .
\end{aligned}
$$

The axial anomaly thus arises in the way suggested long ago by Fujikawa [83]. In particular, the symmetry structure in this formulation of lattice QCD is exactly as expected.

\section{Gauge-invariant lattice regularization of anomaly-free theories}

Vector-like theories with any gauge group and fermion representation can be put on the lattice in the same manner, using the appropriate gauge-covariant version of the lattice Dirac operator (5.11). We can then pass to the associated chiral theory simply by imposing the constraints (5.8) on the fermion and antifermion fields. As far as the field space, the lattice action and the classical field equations are concerned, the projection to the left-handed fields is completely consistent in this case, since it derives from an underlying exact symmetry.

When we now try to quantize this theory through the functional integral, the principal difficulty is that there is a non-trivial phase ambiguity in the fermion integration measure. The problem is related to the gauge anomaly and will occupy us throughout this section. For simplicity we shall focus on the construction of the measure in perturbation theory and shall ignore all complications that have to do with non-perturbative effects such as the global anomalies [6-8]. 
Before plunging into the details, it is worth mentioning that the fifth dimension has completely disappeared at this point. We could in fact start from any gaugecovariant lattice Dirac operator that satisfies the Ginsparg-Wilson relation and a few technical conditions (locality, absence of unphysical poles, etc.). The operator (5.11) has all these properties, and also the "perfect" Dirac operator $[17,84,85]$, which derives from the block-spin renormalization group transformations studied previously by Ginsparg and Wilson in their famous work [57].

\subsection{Functional integral}

As usual the basic quantities to be considered in the quantized theory are correlation functions $\left\langle\phi_{1}\left(x_{1}\right) \ldots \phi_{n}\left(x_{n}\right)\right\rangle$ of gauge-invariant local fields. Formally they are given by the functional integral

$$
\begin{aligned}
& \left\langle\phi_{1}\left(x_{1}\right) \ldots \phi_{n}\left(x_{n}\right)\right\rangle= \\
& \frac{1}{\mathcal{Z}} \int \mathrm{D}[U] \int \mathrm{D}[\psi]_{\text {left }} \mathrm{D}[\bar{\psi}]_{\text {left }} \phi_{1}\left(x_{1}\right) \ldots \phi_{n}\left(x_{n}\right) \mathrm{e}^{-S_{\mathrm{G}}[U]-S_{\mathrm{F}}[U, \bar{\psi}, \psi]},
\end{aligned}
$$

where $\mathrm{D}[U]$ denotes the standard integration measure for lattice gauge fields and the normalization constant $\mathcal{Z}$ is defined through the requirement that $\langle 1\rangle=1$.

Since the measure $\mathrm{D}[U]$ and the gauge-field action $S_{\mathrm{G}}[U]$ will not concern us here, there is no need to specify them explicitly. It suffices to know that they are locally defined and that they preserve the gauge and the lattice symmetries [76,77]. The fermion action (5.20) is also local and invariant so that a problem (if any) can only arise from the integration measures $\mathrm{D}[\psi]_{\text {left }}$ and $\mathrm{D}[\bar{\psi}]_{\text {left }}$.

Let us now have a closer look at the fermion measure. In terms of an orthonormal basis $v_{j}(x)$ of left-handed Dirac fields, the fermion field may be written as

$$
\psi(x)=\sum_{j} v_{j}(x) c_{j}
$$

The coefficients $c_{j}$ in this expansion represent the independent degrees of freedom of the field, and a possible fermion measure is thus given by

$$
\mathrm{D}[\psi]_{\mathrm{left}}=\prod_{j} \mathrm{~d} c_{j}
$$

There is in fact not much choice here, since the coefficients $c_{j}$ are the generators of a Grassmann algebra (we are dealing with fermions). The integration measure on 


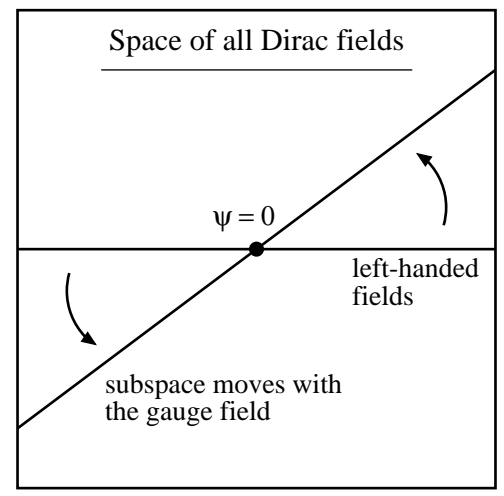

Fig. 6. The projector $\hat{P}_{-}$maps the space of all lattice Dirac fields to the subspace of left-handed fields. Since the projector involves the lattice Dirac operator $D$, the subspace changes when the gauge field is varied.

any such algebra is unique up to a complex proportionality factor. In particular, if we pass to a different orthonormal basis,

$$
\tilde{v}_{j}(x)=\sum_{l} v_{l}(x)\left(\mathcal{Q}^{-1}\right)_{l j}, \quad \tilde{c}_{j}=\sum_{l} \mathcal{Q}_{j l} c_{l},
$$

the measure changes by the factor $\operatorname{det} \mathcal{Q}$, which is a pure phase factor since the transformation matrix $\mathcal{Q}$ is unitary.

The antifermion measure $\mathrm{D}[\bar{\psi}]_{\text {left }}$ is defined in the same way, using a basis $\bar{v}_{k}(x)$ of left-handed fields. An important difference is that the basis can be taken to be independent of the gauge field, while this is not possible in the fermion case, because the subspace of left-handed fermion fields moves with the gauge field (see fig. 6). As a result the fermion measure and the partition function

$$
\mathrm{e}^{-S_{\text {eff }}[U]}=\int \mathrm{D}[\psi]_{\text {left }} \mathrm{D}[\bar{\psi}]_{\text {left }} \mathrm{e}^{-S_{\mathrm{F}}[U, \bar{\psi}, \psi]}
$$

have a gauge-field dependent phase ambiguity. Evidently, the phase matters in the functional integral (6.1), and the theory hence remains incompletely specified at the quantum level until the ambiguity in the measure has been fixed.

Apart from this the structure of the theory has now been completely clarified. In particular, since the integral over the fermion and antifermion fields is gaussian, the 
fermion correlation functions are given by

$$
\begin{gathered}
\int \mathrm{D}[\psi]_{\text {left }} \mathrm{D}[\bar{\psi}]_{\text {left }} \psi\left(x_{1}\right) \ldots \psi\left(x_{n}\right) \bar{\psi}\left(y_{1}\right) \ldots \bar{\psi}\left(y_{n}\right) \mathrm{e}^{-S_{\mathrm{F}}[U, \bar{\psi}, \psi]}= \\
\mathrm{e}^{-S_{\text {eff }}[U]} \times\{\text { sum of Wick contractions }\}
\end{gathered}
$$

The only non-zero two-point contraction is

$$
\overline{\psi(x) \bar{\psi}}(y)=\hat{P}_{-} S(x, y) P_{+},
$$

where $S(x, y)$ denotes the Green function of the lattice Dirac operator in the presence of the gauge field. The fermion integral thus yields the expected result for the chiral propagator (cf. subsect. 5.2).

\subsection{Locality}

The phase of the fermion measure should obviously be such that the locality properties and the symmetries of the theory are preserved. This requirement turns out to be very strong, and it can be shown to determine the phase up to irrelevant local terms (i.e. up to the usual discretization ambiguities) [33-37]. Of course, we may be unable to choose the phase in this way, but it is surely reasonable to try to get there.

We now need to say what locality precisely means in the present context. Directional derivatives in field space are going to play an important rôle in this discussion, and we thus introduce these first. So let

$$
U_{t}(x, \mu)=\mathrm{e}^{\operatorname{ta}_{\mu}(x)} U(x, \mu), \quad \eta_{\mu}(x)=\eta_{\mu}^{a}(x) T^{a},
$$

be a smooth one-parameter family of lattice gauge fields (see fig. 7). We can think of $U_{t}$ as a curve in field space, and if $F[U]$ is any given functional, its rate of change along the curve at the point $U_{0}=U$ is

$$
\delta_{\eta} F[U]=\left\{\frac{\mathrm{d}}{\mathrm{d} t} F\left[U_{t}\right]\right\}_{t=0} .
$$

The differential operator $\delta_{\eta}$, which is defined through this equation, may be regarded as a derivative in the direction of the tangential vector $\eta_{\mu}^{a}(x)$. It is analogous to the derivative

$$
\int \mathrm{d}^{4} x \eta_{\mu}^{a}(x) \frac{\delta}{\delta A_{\mu}^{a}(x)}
$$




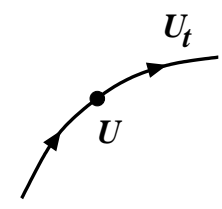

Fig. 7. Equation (6.8) describes a curve in the space of all lattice gauge fields, with curve parameter $t$ and tangential vector $\eta_{\mu}^{a}(x)$ at $t=0$.

in the continuum theory and in fact converges to this operator in the continuum limit.

The derivative of the effective action, $\delta_{\eta} S_{\text {eff }}[U]$, can be worked out by applying $\delta_{\eta}$ on both sides of eq. (6.5). Note that the fermion field in the integral is to be replaced by the expansion (6.2). The fermion action then depends on the gauge field not only through the Dirac operator but also through the basis vectors $v_{j}(x)$. As a result the calculation yields two terms,

$$
\begin{aligned}
& \delta_{\eta} S_{\text {eff }}=-\operatorname{Tr}\left\{\delta_{\eta} D \hat{P}_{-} D^{-1} P_{+}\right\}+i \mathfrak{L}_{\eta}, \\
& \mathfrak{L}_{\eta}=i \sum_{j}\left(v_{j}, \delta_{\eta} v_{j}\right)
\end{aligned}
$$

of which the second characterizes the chosen measure and is hence referred to as the measure term. It depends linearly on the direction $\eta_{\mu}^{a}(x)$, i.e. there exists a current $j_{\mu}^{a}(x)$ such that

$$
\mathfrak{L}_{\eta}=a^{4} \sum_{x} \eta_{\mu}^{a}(x) j_{\mu}^{a}(x)
$$

At this point little is known about this current, except that it is a well-defined function of the gauge field for any given choice of the fermion measure.

If we temporarily consider the gauge field to be an external classical field, eq. (6.11) may be interpreted as the response of the fermion sector to a change of the field. The current $j_{\mu}^{a}(x)$ then appears on the right-hand side of the semiclassical field equations, together with the other term,

$$
-\operatorname{Tr}\left\{\delta_{\eta} D \hat{P}_{-} D^{-1} P_{+}\right\}=a^{4} \sum_{x}\left\langle\bar{\psi}(x) \delta_{\eta} D \psi(x)\right\rangle_{\mathrm{F}},
$$

which can be written as a fermion expectation value of a local operator. A characteristic property of local theories is that the field equations are linear relations 
between local operator insertions. We are thus led to require that the current $j_{\mu}^{a}(x)$ should be a local expression in the gauge field. The measure term in eq. (6.11) then assumes the form of a local counterterm and the theory is only minimally affected by the gauge-field dependence of the fermion measure.

\subsection{Gauge invariance}

The gauge transformation behaviour of the effective action can be studied by computing its variation along the gauge directions in field space. From the transformation law (3.10) it follows that these directions are given by

$$
\eta_{\mu}(x)=-\nabla_{\mu} \omega(x), \quad \omega(x)=\omega^{a}(x) T^{a},
$$

where $\omega^{a}(x)$ is an arbitrary lattice field, transforming according to the adjoint representation of the gauge group, and $\nabla_{\mu}$ the appropriate covariant difference operator (cf. subsect. 3.3).

The gauge variation of the effective action may now be worked out by inserting eq. (6.15) in eq. (6.11). Using the gauge covariance of the Dirac operator, a few lines of algebra then lead to the result

$$
\begin{aligned}
& \delta_{\eta} S_{\mathrm{eff}}=i a^{4} \sum_{x} \omega^{a}(x)\left\{\left[\nabla_{\mu}^{*} j_{\mu}\right]^{a}(x)-\mathcal{A}^{a}(x)\right\}, \\
& \mathcal{A}^{a}(x)=\frac{1}{2} i a \operatorname{tr}\left\{R\left(T^{a}\right) \gamma_{5} D(x, x)\right\} .
\end{aligned}
$$

Since the kernel $D(x, y)$ of the Dirac operator depends locally on the gauge field [21], it is obvious that $\mathcal{A}^{a}(x)$ is a gauge-covariant local composite field. In the continuum limit we have $[22-27,35]$

$$
\mathcal{A}^{a}(x) \underset{a \rightarrow 0}{\sim}-\frac{1}{128 \pi^{2}} d_{R}^{a b c} \epsilon_{\mu \nu \rho \sigma} F_{\mu \nu}^{b}(x) F_{\rho \sigma}^{c}(x)+\mathrm{O}(a),
$$

and $\mathcal{A}^{a}(x)$ may hence be regarded as a lattice representation of the so-called covariant gauge anomaly $[1,2]$.

To ensure the gauge invariance of the effective action (and consequently of the full theory), the right-hand side of eq. (6.16) must vanish for all gauge variations. The fermion measure should hence be such that the associated current $j_{\mu}^{a}(x)$ satisfies

$$
\left[\nabla_{\mu}^{*} j_{\mu}\right]^{a}(x)=\mathcal{A}^{a}(x) .
$$

Since the anomaly is a local field, this is consistent with the requirement of locality, although there is obviously no guarantee that both conditions can be met. 


\subsection{Integrability condition}

Apart from locality and gauge invariance, there is a further constraint on the current $j_{\mu}^{a}(x)$ that derives from the fact that eq. (6.11) must be integrable. In other words, if the equation is integrated along any smooth path $U_{t}$ in field space, the integral should only depend on the initial and final field configurations, but not on the shape of the curve. This will be the case if and only if the curl in field space of the right-hand side of eq. (6.11) vanishes.

Using the identities (5.6), this leads to the equation

$$
\delta_{\eta} \mathfrak{L}_{\zeta}-\delta_{\zeta} \mathfrak{L}_{\eta}+a \mathfrak{L}_{[\eta, \zeta]}=i \operatorname{Tr}\left\{\hat{P}_{-}\left[\delta_{\eta} \hat{P}_{-}, \delta_{\zeta} \hat{P}_{-}\right]\right\}
$$

where the commutator of the two vector fields $\eta_{\mu}^{a}(x)$ and $\zeta_{\mu}^{a}(x)$ is to be taken pointwise. Note that the right-hand side of eq. (6.20) is a known local function of these fields and the gauge field, while the other side involves the measure term only. The integrability condition thus has the form of an inhomogeneous partial differential equation in field space for the current $j_{\mu}^{a}(x)$.

So far the fermion measure has been taken as the primary quantity from which the associated current is obtained. We can now invert this relationship since any given current that satisfies the integrability condition arises from an underlying fermion measure [35]. In other words, to complete the construction of the theory, it suffices to find a current $j_{\mu}^{a}(x)$ that (a) is a local expression in the gauge field, (b) fulfils the requirement of gauge invariance, eq. (6.19), and (c) solves the integrability condition (6.20). This does not look like an easy task, but the problem is in fact much more accessible than it seems to be, at least in perturbation theory.

\subsection{Construction of the fermion measure in perturbation theory}

The perturbation expansion of the functional integral (6.1) around the vacuum configuration $U(x, \mu)=1$ is obtained essentially as in lattice QCD. An explicit specification of the fermion measure is not required if we first integrate over the fermion fields using eqs. (6.6),(6.7). The integral then assumes the form

$$
\left\langle\phi_{1}\left(x_{1}\right) \ldots \phi_{n}\left(x_{n}\right)\right\rangle=\frac{1}{\mathcal{Z}} \int \mathrm{D}[U]\left\langle\phi_{1}\left(x_{1}\right) \ldots \phi_{n}\left(x_{n}\right)\right\rangle_{\mathrm{F}} \mathrm{e}^{-S_{\mathrm{G}}[U]-S_{\mathrm{eff}}[U]}
$$

As usual the gauge needs to be fixed and the lattice field $U(x, \mu)$ is parametrized through a gauge potential $A_{\mu}^{a}(x)$ according to eq. (3.13). After rescaling

$$
A_{\mu}^{a}(x) \rightarrow g_{0} A_{\mu}^{a}(x)
$$


(where $g_{0}$ denotes the bare gauge coupling), the perturbation series is obtained by expanding the integrand in eq. (6.21) in powers of $g_{0}$.

The only really new element here is the measure term, which [via eq. (6.11)] contributes to the expansion of the effective action. In perturbation theory the associated current is given by

$$
j_{\mu}^{a}(x)=\sum_{n=0}^{\infty} \frac{g_{0}^{n}}{n !} a^{4 n} \sum_{x_{1}, \ldots, x_{n}} L^{(n)}\left(x, x_{1}, \ldots, x_{n}\right)_{\mu \mu_{1} \ldots \mu_{n}}^{a a_{1} \ldots a_{n}} A_{\mu_{1}}^{a_{1}}\left(x_{1}\right) \ldots A_{\mu_{n}}^{a_{n}}\left(x_{n}\right),
$$

and we are now left with the problem of determining the coefficients $L^{(n)}$ so that the conditions listed above are satisfied. The expansion of the effective action can then be worked out straightforwardly by repeated differentiation of eq. (6.11) with respect to the gauge potential.

It may be useful at this point to briefly state what the conditions (a)-(c) mean in terms of the coefficients $L^{(n)}$. To fulfil the requirement of locality, the coefficients must be translation-invariant and exponentially decreasing if any of the distances $\left\|x_{k}-x\right\|$ becomes larger than a few lattice spacings. The other conditions both turn into towers of inhomogeneous linear equations for the coefficients once the anomalous conservation law (6.19) and the integrability condition (6.20) are expanded in powers of the gauge potential. Although this rapidly becomes very tedious, it is possible to work out the right-hand sides of these equations explicitly by expressing them through the kernel $D(x, y)$ of the Dirac operator and expanding the latter following refs. [22,37,86-89].

It is surely far from obvious that all these equations can be solved, but it turns out that a solution can be constructed through a purely algebraic recursive procedure [37]. As a result we have the following

Theorem. If the fermion representation $R$ is anomaly-free, there exist lattice functions $L^{(n)}$ such that

(1) $j_{\mu}^{a}(x)$ satisfies conditions (a)-(c) to all orders of $g_{0}$,

(2) $L^{(n)}=0$ for $n \leq 3$,

(3) $\operatorname{Im} S_{\text {eff }}$ transforms like a pseudoscalar under the lattice symmetries.

While there is more than one solution with these properties, the difference between any two of them amounts to a change of the effective action by a term of the form

$$
\Delta S_{\text {eff }}[U]=a^{4} \sum_{x} \Omega(x)
$$


where $\Omega(x)$ is a gauge-invariant, pseudoscalar local field of dimension greater than or equal to 5 . For dimensional reasons, $\Omega(x)$ must be proportional to a positive power of the lattice spacing, and up to finite renormalizations such differences are hence not expected to have any influence on the theory in the continuum limit.

At this point the lattice theory has been completely specified, to all orders of the gauge coupling. The fact that we have been able to meet all conditions on the fermion measure shows that anomaly-free chiral gauge theories can be consistently regularized without breaking the gauge symmetry. While the construction of this regularization is obviously non-trivial, it should be emphasized that no computation of Feynman diagrams was required. The equations that had to be solved are explicitly given and involve local expressions only.

\subsection{Anomaly cancellation 8 cohomology}

The demonstration of the exact cancellation of the gauge anomaly on the lattice is perhaps the most difficult step in the proof of the theorem quoted above. We shall not attempt to describe this in any detail, but a quick look at the problem and its solution in the abelian case may be illuminating.

So let us consider a $U(1)$ theory with fermion representation (2.16). Since there is only one group generator, the anomaly (6.17) has no index and is given by

$$
\mathcal{A}(x)=\frac{1}{2} i a \operatorname{tr}\left\{R \gamma_{5} D(x, x)\right\}, \quad R=i \times \operatorname{diag}\left(\mathrm{e}_{1}, \ldots, \mathrm{e}_{N}\right) .
$$

In the present context we may assume that $\mathcal{A}(x)$ is expanded in powers of the gauge potential $A_{\mu}(x)$ similarly to the current $j_{\mu}(x)$ [eq. (6.23)]. From eq. (6.25) we then infer that the associated coefficients are local and that the series is invariant under arbitrary gauge transformations

$$
A_{\mu}(x) \rightarrow A_{\mu}(x)+g_{0}^{-1} \partial_{\mu} \omega(x)
$$

(where $\partial_{\mu}$ denotes the forward difference operator).

A less obvious property of the anomaly is that

$$
a^{4} \sum_{x} \delta \mathcal{A}(x)=0
$$

for any local variation $\delta A_{\mu}(x)$ of the gauge potential. To prove this we note that the left-hand side of eq. (6.27) is proportional to $\operatorname{Tr}\left\{R \delta \hat{\gamma}_{5}\right\}$. We then insert $\left(\hat{\gamma}_{5}\right)^{2}=1$ and cycle one of the factors $\hat{\gamma}_{5}$ around the trace using

$$
\left\{\hat{\gamma}_{5}, \delta \hat{\gamma}_{5}\right\}=0, \quad\left[\hat{\gamma}_{5}, R\right]=0 .
$$


As a result the trace is reproduced with the opposite sign and it must hence be equal to zero.

Equation (6.27) says that the abelian anomaly is a local topological field, i.e. it has all the characteristic properties of a topological density. Modulo divergence terms (which are topologically uninteresting) there are usually not many fields of this type, and the form of the anomaly is thus strongly constrained. The classification of topological fields is a particular case of a local cohomology problem, a subject that has received a lot of attention in continuum field theory. In particular, using the descent equations [39-43], a general theorem has been established which states that, in pure gauge theories with any gauge group, the Chern monomials are the only non-trivial topological fields [90,91].

On the lattice, a similar classification theorem holds for $\mathrm{U}(1)$ theories [30,31]. Any topological field $q(x)$ can be shown to be of the form

$$
q(x)=c(x)+\partial_{\mu}^{*} k_{\mu}(x)
$$

in this case, where $k_{\mu}(x)$ is a gauge-invariant local current and

$$
\begin{aligned}
& c(x)=\alpha+\beta_{\mu \nu} F_{\mu \nu}(x)+\gamma \epsilon_{\mu \nu \rho \sigma} F_{\mu \nu}(x) F_{\rho \sigma}(x+a \hat{\mu}+a \hat{\nu}), \\
& F_{\mu \nu}(x)=\partial_{\mu} A_{\nu}(x)-\partial_{\nu} A_{\mu}(x),
\end{aligned}
$$

the general Chern polynomial on the lattice $\dagger$. The proof of the theorem is constructive in the sense that the coefficients of the current $k_{\mu}(x)$ are obtained algebraically in terms of the coefficients of $q(x)$.

In the case of the anomaly, the constants $\alpha$ and $\beta_{\mu \nu}$ must vanish, because $\mathcal{A}(x)$ transforms like a pseudoscalar field under the lattice symmetries. Concerning the constant $\gamma$ we note that the anomaly is a sum of terms, one for each fermion flavour. Since the field tensor scales with the charge and since there is another power of the charge coming from the representation matrix $R$ in eq. (6.25), we have

$$
\gamma \propto \sum_{\alpha=1}^{N} \mathrm{e}_{\alpha}^{3}
$$

$\dagger$ In the last term in eq. (6.30) the coordinate shift is required to ensure that $c(x)$ is topological. Its presence can be traced back to the fact that the difference operators $\partial_{\mu}$ and $\partial_{\mu}^{*}$ obey a modified Leibniz rule [31]. 


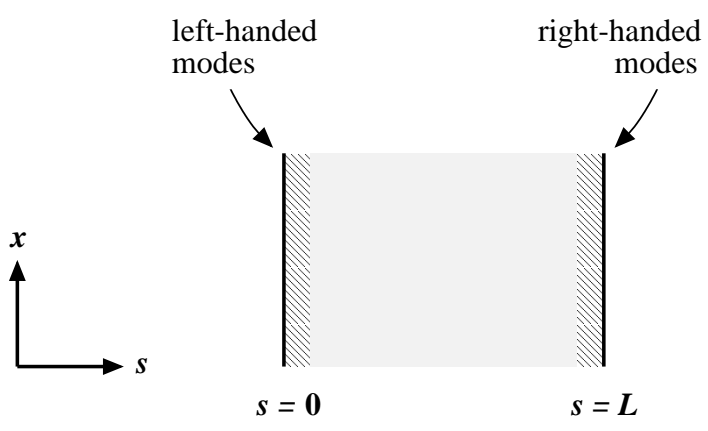

Fig. 8. At low energies, massive fermions in a five-dimensional volume with two boundaries reduce to left- and right-handed fermions in four dimensions that couple to the gauge field in the vicinity of the boundaries.

The topologically non-trivial part of the anomaly thus cancels if the fermion representation is anomaly-free, and the condition for exact gauge invariance, eq. (6.19), then reduces to

$$
\partial_{\mu}^{*} j_{\mu}(x)=\partial_{\mu}^{*} k_{\mu}(x)
$$

Although more work is required to actually show this [33,37], it should now be quite plausible that a current $j_{\mu}(x)$ can be found that satisfies this equation and all the other conditions. At least the topological obstruction represented by the anomaly has completely disappeared at this stage.

\subsection{Epilogue}

In order to bring out the basic ideas as clearly as possible, many technical issues have been left aside in this section and the presentation has often been rather abbreviated. A much more detailed description of the formulation of chiral lattice gauge theories in perturbation theory, to the extent that explicit computations could start from there, can be found in ref. [37].

At the non-perturbative level a general construction of the fermion measure is still missing. The principal difficulties are that there can be global obstructions and that the local constraints are less accessible if the equations may not be expanded in powers of the gauge potential [35]. Currently all these mathematical problems have been completely solved only for abelian gauge groups [33].

In these lectures we made a long excursion to five dimensions, which led us to the Ginsparg-Wilson relation and a new realization of chiral symmetry. This proved to be the key to the construction of chiral lattice gauge theories in four dimensions. 
The question may now be asked whether chiral gauge theories can also be obtained directly from a field theory in five (or more) dimensions. We might consider a fivedimensional volume with two boundaries, for example, and couple an $s$-dependent gauge field $U(x, s, \mu)$ to a massive Dirac field (see fig. 8). There are massless fermion modes with both chiralities in this case, which interact with the gauge field in the vicinity of the boundaries. So if we impose the boundary conditions

$$
\left.U(x, s, \mu)\right|_{s=0}=U(x, \mu),\left.\quad U(x, s, \mu)\right|_{s=L}=1,
$$

the right-handed modes are expected to decouple for large wall separations $L$, while the left-handed modes couple to the physical gauge field $U(x, \mu)$ only.

This is in fact what happens if the gauge field is treated as an external classical field and if the fermion representation is anomaly-free [50-56]. For several reasons it remains unclear, however, whether a positive answer to the question posed above can be given along these lines. The main problem at present is that the gauge field in the bulk of the five-dimensional volume needs to be decoupled from the physical field at the boundary, and there seems to be no natural way to achieve this.

\section{References}

[1] L. Alvarez-Gaumé, An introduction to anomalies, in: Fundamental problems of gauge field theory (Erice 1985), eds. G. Velo, A. S. Wightman (Plenum Press, New York, 1986)

[2] R. A. Bertlmann, Anomalies in quantum field theory (Oxford University Press, Oxford, 1996)

[3] C. Becchi, A. Rouet, R. Stora, Commun. Math. Phys. 42 (1975) 127; Ann. Phys. (NY) 98 (1976) 287

[4] C. Becchi, Lectures on the renormalization of gauge theories, in: Relativity, groups and topology (Les Houches 1983), eds. B. S. DeWitt, R. Stora (North-Holland, Amsterdam, 1984)

[5] O. Piguet, S. P. Sorella, Algebraic renormalization: perturbative renormalization, symmetries and anomalies, Lecture Notes in Physics m28 (Springer-Verlag, Berlin, 1995)

[6] E. Witten, Phys. Lett. B117 (1982) 324; Nucl. Phys. B223 (1983) 422

[7] S. Elitzur, V. P. Nair, Nucl. Phys. B243 (1984) 205

[8] O. Bär, I. Campos, Nucl. Phys. B (Proc. Suppl.) 83-84 (2000) 594; Nucl. Phys. B581 (2000) 499

[9] A. Borrelli, L. Maiani, G. C. Rossi, R. Sisto, M. Testa, Phys. Lett. B221 (1989) 360; Nucl. Phys. B333 (1990) 335 
[10] M. Testa, The Rome approach to chirality, in: Recent developments in non-perturbative quantum field theory (Seoul 1997), eds. Y. M. Cho, M. Virasoro (World Scientific, Singapore, 1998)

[11] J. L. Alonso, J. L. Cortes, E. Rivas, Int. J. Mod. Phys. A5 (1990) 2839

[12] J. L. Alonso, P. Boucaud, F. Lesmes, A. J. van der Sijs, Nucl. Phys. B457 (1995) 175

[13] W. Bock, M. F. L. Golterman, Y. Shamir, Nucl. Phys. (Proc. Suppl.) 63 (1998) 147 and 581; Phys. Rev. Lett. 80 (1998) 3444; Phys. Rev. D58 (1998) 034501

[14] W. Bock, M. F. L. Golterman, K. C. Leung, Y. Shamir, Nucl. Phys. B (Proc. Suppl.) 83-84 (2000) 603; Chin. J. Phys. 38 (2000) 647

[15] M. Testa, Chin. J. Phys. 38 (2000) 563

[16] P. A. Grassi, T. Hurth, hep-ph/0101183

[17] P. Hasenfratz, Nucl. Phys. B (Proc. Suppl.) 63A-C (1998) 53; Nucl. Phys. B525 (1998) 401

[18] H. Neuberger, Phys. Lett. B417 (1998) 141; ibid. B427 (1998) 353

[19] P. Hasenfratz, V. Laliena, F. Niedermayer, Phys. Lett. B427 (1998) 125

[20] M. Lüscher, Phys. Lett. B428 (1998) 342

[21] P. Hernández, K. Jansen, M. Lüscher, Nucl. Phys. B552 (1999) 363

[22] Y. Kikukawa, A. Yamada, Phys. Lett. B448 (1999) 265

[23] T.-W. Chiu, Phys. Lett. B445 (1999) 371

[24] T. Reisz, H. J. Rothe, Phys. Lett. B455 (1999) 246; Nucl. Phys. B575 (2000) 255

[25] K. Fujikawa, Nucl. Phys. B546 (1999) 480

[26] H. Suzuki, Prog. Theor. Phys. 102 (1999) 141

[27] D. H. Adams, hep-lat/9812003; Nucl. Phys. B589 (2000) 633

[28] R. Narayanan, Phys. Rev. D58 (1998) 97501

[29] F. Niedermayer, Nucl. Phys. B (Proc. Suppl.) 73 (1999) 105

[30] M. Lüscher, Nucl. Phys. B538 (1999) 515

[31] T. Fujiwara, H. Suzuki, K. Wu, Phys. Lett. B463 (1999) 63; Nucl. Phys. B569 (2000) 643

[32] Y. Kikukawa, Y. Nakayama, hep-lat/0005015

[33] M. Lüscher, Nucl. Phys. B549 (1999) 295

[34] H. Suzuki, Prog. Theor. Phys. 101 (1999) 1147

[35] M. Lüscher, Nucl. Phys. B568 (2000) 162

[36] H. Suzuki, Nucl. Phys. B585 (2000) 471 [E: H. Igarashi, K. Okuyama, H. Suzuki, heplat/0012018

[37] M. Lüscher, J. High Energy Phys. 06 (2000) 028

[38] H. Suzuki, J. High Energy Phys. 10 (2000) 039

[39] R. Stora, Continuum gauge theories, in: New developments in quantum field theory and statistical mechanics (Cargèse 1976), eds. M. Lévy, P. Mitter (Plenum Press, New York, 1977)

[40] R. Stora, Algebraic structure and topological origin of anomalies, in: Progress in gauge field theory (Cargèse 1983), eds. G. 't Hooft et al. (Plenum Press, New York, 1984) 
[41] B. Zumino, Chiral anomalies and differential geometry, in: Relativity, groups and topology (Les Houches 1983), eds. B. S. DeWitt, R. Stora (North-Holland, Amsterdam, 1984)

[42] L. Baulieu, Algebraic construction of gauge invariant theories, in: Particles and Fields (Cargèse 1983), eds. M. Lévy et al. (Plenum, New York, 1985)

[43] L. Baulieu, Nucl. Phys. B241 (1984) 557; Phys. Rep. 129 (1985) 1

[44] V. Rubakov, M. Shaposhnikov, Phys. Lett. B125 (1983) 136

[45] C. G. Callan, J. A. Harvey, Nucl. Phys. B250 (1985) 427

[46] D. B. Kaplan, Phys. Lett. B288 (1992) 342; Nucl. Phys. B (Proc. Suppl.) 30 (1993) 597

[47] Y. Shamir, Nucl. Phys. B406 (1993) 90

[48] V. Furman, Y. Shamir, Nucl. Phys. B439 (1995) 54

[49] Y. Kikukawa, T. Noguchi, hep-lat/9902022

[50] L. Alvarez-Gaumé, S. Della Pietra, V. Della Pietra, Phys. Lett. B166 (1986) 177; Commun. Math. Phys. 109 (1987) 691

[51] L. Alvarez-Gaumé, S. Della Pietra, The effective action for chiral fermions, in: Recent developments in quantum field theory (Niels Bohr Centennial Conference, Copenhagen, 1985), eds. J. Ambjørn et al. (North-Holland, Amsterdam, 1985)

[52] R. D. Ball, H. Osborn, Phys. Lett. B165 (1985) 410; Nucl. Phys. B263 (1986) 245

[53] R. D. Ball, Phys. Lett. B171 (1986) 435; Phys. Rep. 182 (1989) 1

[54] D. B. Kaplan, M. Schmaltz, Phys. Lett. B368 (1996) 44

[55] H. Suzuki, Prog. Theor. Phys. 101 (1999) 1147

[56] T. Aoyama, Y. Kikukawa, hep-lat/9905003

[57] P. H. Ginsparg, K. G. Wilson, Phys. Rev. D25 (1982) 2649

[58] Y. Shamir, Nucl. Phys. B (Proc. Suppl.) 47 (1996) 212

[59] M. Lüscher, Nucl. Phys. B (Proc. Suppl.) 83-84 (2000) 34

[60] H. Neuberger, Nucl. Phys. B (Proc. Suppl.) 83-84 (2000) 67

[61] M. F. L. Golterman, hep-lat/0011027

[62] H. Leutwyler, Phys. Lett. B152 (1985) 78

[63] H. Georgi, S. L. Glashow, Phys. Rev. Lett. 32 (1974) 438

[64] D. P. Želobenko, Compact Lie groups and their representations (American Mathematical Society, Providence, 1973)

[65] G. 't Hooft, M. Veltman, Nucl. Phys. B44 (1972) 189

[66] A. A. Slavnov, Nucl. Phys. B31 (1971) 301

[67] B. W. Lee, J. Zinn-Justin, Phys. Rev. D5 (1972) 3121

[68] A. A. Slavnov, Theor. Math. Phys. 33 (1977) 977

[69] L. D. Faddeev, A. A. Slavnov, Gauge fields: introduction to quantum theory, 2nd ed. (Benjamin-Cummings, London, 1991)

[70] C. P. Martin, F. Ruiz Ruiz, Nucl. Phys. B436 (1995) 545

[71] J. H. León, C. P. Martin, F. Ruiz Ruiz, Phys. Lett. B355 (1995) 531

[72] M. Asorey, F. Falceto, Phys. Rev. D54 (1996) 5290 
[73] T. D. Bakeyev, A. A. Slavnov, Mod. Phys. Lett. A11 (1996) 1539

[74] M. M. Deminov, A. A. Slavnov, hep-th/0012138

[75] K. G. Wilson, Phys. Rev. D10 (1974) 2445

[76] I. Montvay, G. Münster, Quantum fields on a lattice (Cambridge University Press, Cambridge, 1994)

[77] H. J. Rothe, Lattice gauge theories: an introduction, 2nd ed. (World Scientific, Singapore, 1997)

[78] M. Lüscher, Selected topics in lattice field theory, in: Fields, strings and critical phenomena (Les Houches 1988), eds. E. Brézin, J. Zinn-Justin (North-Holland, Amsterdam, 1989)

[79] T. Reisz, Commun. Math. Phys. 116 (1988) 81 and 573; ibid. 117 (1988) 79 and 639; Nucl. Phys. B318 (1989) 417

[80] M. Lüscher, P. Weisz, Nucl. Phys. B452 (1995) 213

[81] H. B. Nielsen, M. Ninomiya, Phys. Lett. B105 (1981) 219; Nucl. Phys. B185 (1981) 20 [E: ibid. B195 (1982) 541]; ibid. B193 (1981) 173

[82] D. Friedan, Commun. Math. Phys. 85 (1982) 481

[83] K. Fujikawa, Phys. Rev. Lett. 42 (1979) 1195; Phys. Rev. D21 (1980) 2848 [E: ibid. D22 (1980) 1499]

[84] P. Hasenfratz, F. Niedermayer, Nucl. Phys. B414 (1994) 785

[85] P. Hasenfratz, The theoretical background and properties of perfect actions, in: Nonperturbative quantum field physics (Peñiscola 1997), eds. M. Asorey, A. Dobado (World Scientific, Singapore, 1998)

[86] C. Alexandrou, H. Panagopoulos, E. Vicari, Nucl. Phys. B571 (2000) 257

[87] M. Ishibashi, Y. Kikukawa, T. Noguchi, A. Yamada, Nucl. Phys. B576 (2000) 501

[88] C. Alexandrou, E. Follana, H. Panagopoulos, E. Vicari, Nucl. Phys. B580 (2000) 394

[89] S. Capitani, Nucl. Phys. B592 (2000) 183

[90] F. Brandt, N. Dragon, M. Kreuzer, Phys. Lett. B231 (1989) 263; Nucl. Phys. B332 (1990) 224 and 250

[91] M. Dubois-Violette, M. Henneaux, M. Talon, C.-M. Viallet, Phys. Lett. B267 (1991) 81; ibid. B289 (1992) 361 